\title{
Diversidad de ácaros (Acari: Prostigmata, Mesotigmata, Astigmata) asociados a la hojarasca de formaciones vegetales del Parque Universitario de la UCLA, Venezuela
}

\author{
Carlos Vásquez ${ }^{1}$ Carlos Sánchez² \& Neicy Valera ${ }^{1}$
}

1. Universidad Centroccidental Lisandro Alvarado (UCLA, Decanato de Agronomía. Departamento de Ciencias Biológicas), Edif. Rectorado, carrera 19 con calle 9, Barquisimeto, Lara, Venezuela. (carlosvasquez@ucla.edu.ve)

2. Ministerio del Ambiente y los Recursos Naturales (MARN, Dirección General de Cuencas Hidrográficas, Dirección de Suelos), Centro Simón Bolívar, Torre Sur, piso 5, Caracas, Distrito Capital, Venezuela. (carlsanz@yahoo.com)

\begin{abstract}
Mite diversity (Acari: Prostigmata, Mesotigmata, Astigmata) associated to soil litter from two vegetation zones at the University Park UCLA, Venezuela. Soil mite diversity (Prostigmata, Mesostigmata, Astigmata) inhabiting in litter-soil layer from a Shrubland and a Deciduous Forest was estimated from December 2000 to November 2001 in the University Park of UCLA (Universidad Centroccidental Lisandro Alvarado), state of Lara, Venezuela. Samplings were made by using pitfall traps along an 1,800 mtransect in both areas. All morfotypes were mounted in microscope slides using Hoyer's medium. A total of 51 mite morfotypes were collected with higher abundance in Prostigmata (2,528) as compared to Mesostigmata (926) and Astigmata (12). Eupodidae (Prostigmata) and Macrochelidae (Mesotigmata) showed higher abundance in both locations. Higher morfotype richness $(S=43)$, diversity $\left(H^{\prime}=2.67\right)$ and evenness $(E=0.69)$ were found in Deciduous Forest compared to values from Shrubland $\left(S=36, H^{\prime}=2.12\right.$ and $\left.E=0.52\right)$. Both areas showed a similarity index $\left(\mathrm{J}^{\prime}\right)$ of 0.59 . Although more individuals were found in Shrubland, the diversity-dominance curves indicated that this is a more disturbed habitat than Deciduous Forest. Further detailed studies are required to determine acarofauna role in trophic web maintenance in soils from tropical areas.
\end{abstract}

KEYWORDS. Diversity, richness, deciduous forest, shrubland, soil mites.

RESUMEN. Se estudió la diversidad de los ácaros (Prostigmata, Mesostigmata y Astigmata) habitantes del estrato suelo-hojarasca de un Matorral y un Bosque Deciduo del Parque Universitario de la UCLA (Universidad Centroccidental Lisandro Alvarado), Estado Lara, Venezuela, desde diciembre 2000 a noviembre 2001. El muestreo se realizó usando trampas de caída colocadas a lo largo de una transecta de $1.800 \mathrm{~m}$ en ambas localidades. Todos los morfotipos fueron montados en láminas microscópicas usando líquido de Hoyer. Se registró un total de 51 morfotipos de ácaros con predominancia del número de individuos pertenecientes a Prostigmata (2528) respecto a Mesostigmata (926) y Astigmata (12). En ambas localidades, Eupodidae (Prostigmata) y Macrochelidae (Mesotigmata) fueron las familias más abundantes. Se determinó mayor riqueza $(S=43)$, diversidad $\left(H^{\prime}=2,67\right)$ y uniformidad $(E=0,69)$ de morfotipos en el Bosque Deciduo al ser comparado con los valores obtenidos en el Matorral $\left(S=36, H^{\prime}=2,12\right.$ y $\left.E=0,52\right)$. Ambas zonas mostraron un índice de similitud (J') de 0,59. La pendiente de la curva de diversidad-dominancia permitió inferir que el Matorral constituye un hábitat más disturbado que el Bosque Deciduo a pesar de albergar mayor número de individuos. Se requiere realizar estudios más detallados que sirvan de base para determinar el rol de la acarofauna en el equilibrio de la red trófica de suelo de las regiones tropicales.

PALABRAS CLAVE. Diversidad, riqueza, bosque deciduo, matorral, ácaros del suelo.

En los ecosistemas terrestres, la hojarasca del suelo constituye la principal fuente de energía para la extraordinaria diversidad de las comunidades de organismos que habitan en el suelo, las cuales están conectados por interacciones altamente complejas (HätTENSCHWILER et al., 2005). Adicionalmente, el tipo y calidad de la hojarasca pueden estar positivamente correlacionados con la riqueza de plantas, la cual proporciona una mayor cantidad de alimento y microhábitats para los microartrópodos del suelo favoreciendo así su diversidad y abundancia (Anderson, 1978; Prieto et al., 2005). Entre los organismos habitantes del suelo, los ácaros y los colémbolos pueden representar el 95\% de los microartrópodos (NeHER \& BARBERCHECK, 1999; Vu \& Nguyen, 2000; Brown \& Fragoso, 2003), encontrándose densidades desde 50.000 hasta 250.000 ácaros $/ \mathrm{m}^{2}$ en los primeros $10 \mathrm{~cm}$ de suelo, por lo que se considera al estrato suelo-hojarasca como su hábitat más ancestral y es allí donde puede encontrarse la mayor diversidad y abundancia (Petersen, 1982; Walter \& Proctor, 1999), sobre todo en ambientes de las regiones tropicales (GASTON, 1992).
Con relación a los ácaros, estudios previos han demostrado que Cryptostigmata (=Oribatida) constituye el grupo más diverso y abundante en el horizonte orgánico del suelo, seguido de Prostigmata (=Actinedida) y Mesostigmata (=Gamasida) (NEHER \& BARBERCHECK, 1999). Los Astigmata (=Acaridida) son poco comunes y generalmente los géneros más encontrados son Rhizoglyphus (Claparède, 1869) y Tyrophagus Oudemans, 1924 (Mineiro \& Moraes, 2002). Los ácaros, junto con otros organismos, juegan un papel relevante en el mantenimiento del equilibrio del ecosistema del suelo, debido al efecto de depredación que ejercen sobre otros artrópodos, además de su participación en la descomposición y ciclaje de nutrientes mediante la transformación de la materia orgánica (Vu \& NGUYeN, 2000). Por otra parte, algunos grupos de ácaros Gamasina han sido considerados como indicadores de los estadios sucesionales sobre desechos industriales presentes en el suelo (Madej \& Skubala, 2002).

A pesar de su importancia, en Venezuela la información disponible sobre la acarofauna del suelo ha 
sido dirigida sólo al estudio de la taxocenosis, principalmente de Cryptostigmata (BALOGH, 1972; BALOGH \& Balogh, 1988, 1990; Behan-Pelletier et al., 1993), mientras que el conocimiento sobre el resto de los grupos de ácaros ha sido limitado al registro de la abundancia de los taxa superiores (órdenes) en agroecosistemas (BRACHO et al., 1999) y en ecosistemas naturales (PAOLETTI etal., 1988, 1991).

Similar a otros ecosistemas naturales de la región tropical, en el Parque Universitario de la UCLA, ubicado dentro los límites del Parque Nacional Terepaima, se encuentran diferentes tipos de vegetación que sirven de refugio y alimentación a una amplia diversidad animal, sin embargo, esta no ha sido suficientemente estudiada. Los pocos estudios sobre los ácaros de Venezuela han estado relacionados con la dificultad para la identificación debido a la amplia diversidad de este grupo, junto con la falta de claves taxonómicas para la identificación de la mesofauna del suelo de la América tropical. Considerando la carencia de información sobre el grupo de los ácaros asociados a la hojarasca en ambientes naturales de Venezuela, en el presente estudio se evaluó la diversidad de familias y géneros de ácaros de los órdenes Prostigmata, Mesostigmata y Astigmata asociados a hojarasca en dos formaciones vegetales (Matorral y Bosque Deciduo) del Parque Universitario de la UCLA.

\section{MATERIALES Y MÉTODOS}

El estudio se realizó en el Parque Universitario "XXV Aniversario de la UCLA" (69¹6'24" - 69¹8'16"E y 9'59'30” - 1001'31'N), Universidad Centroccidental "Lisandro Alvarado" (UCLA), Decanato de Agronomía, Municipio Palavecino, Estado Lara. La zona de estudio presenta una temperatura media anual de $25,1{ }^{\circ} \mathrm{C}$ y precipitación de $658,3 \mathrm{~mm}$ concentrada en régimen unimodal (LóPEZ, 1995) y corresponde con una zona de vida en transición entre un Bosque Muy Seco (BMS) y Bosque Seco (BS) (Holdridge, 1979).

Para el muestreo se estableció una transecta de $1.800 \mathrm{~m}$ dispuesta a lo largo de dos zonas de muestreo delimitadas por el tipo de vegetación (Matorral y Bosque Deciduo). En el Matorral se encuentran suelos de pendientes menores a $30 \%$ y la vegetación dominante está constituida principalmente por arbustos: Zanthoxylum pterota (Rutaceae), Guapira ferruginea (Nyctaginaceae) y Syderoxylon obtusifolium (Sapotaceae), de menos de 3,5 m de altura, y plantas herbáceas: Hyptis suaveolens (Lamiaceae) y Krameria ixine (Krameariaceae). En el Bosque Deciduo se diferencian dos estratos de vegetación; el primero con predominio de árboles deciduos de $7 \mathrm{~m}$ pertenecientes a las especies Lonchocarpus heptaphyllus y Lonchocarpus dipteroneurus (Leguminosae) y el segundo, conformado principalmente por Furcraea humbodltiana (Agavaceae) de $2 \mathrm{~m}$ de altura.

En cada zona de muestreo fueron colocadas 20 trampas de caída con formalina 5\%, las cuales fueron distribuidas en zig-zag a $20 \mathrm{~m}$ de distancia (dependiendo de la topografía del terreno). Las evaluaciones fueron hechas cada 15 días durante el período diciembre 2000 noviembre 2001. En el campo, para separar el líquido de los especimenes atrapados, el contenido de las trampas fue tamizado usando papel de filtro $\mathrm{N}^{\circ} 4$. Posteriormente cada muestra fue colocada individualmente en bolsas de cierre hermético previamente etiquetadas con datos de número de trampa, localidad y fecha de muestreo y llevadas al laboratorio. En el laboratorio, las muestras fueron preservadas en alcohol $70 \%$ y posteriormente los ácaros de los órdenes Prostigmata, Mesostigmata y Astigmata fueron separados bajo magnificación de un microscopio estereoscópico. Los especimenes correspondientes a cada orden fueron transferidos a líquido Koenike con el fin de preservarlos en condiciones óptimas para los futuros estudios (KRANTZ, 1978).

Seguidamente, se prepararon láminas permanentes de cada morfotipo usando líquido de Hoyer, mientras que los ácaros Trombidiformes o muy esclerotizados fueron preparados siguiendo la metodología recomendada por HERDERSON (2001). Las láminas microscópicas fueron secadas en estufa a $45^{\circ} \mathrm{C}$ durante 5 días para su correspondiente identificación. Los ejemplares de cada morfotipo encontrado fueron depositados en la Colección de Acarología del Laboratorio de Zoología Agrícola, UCLA.

Las determinaciones taxonómicas fueron realizadas por los proprios autores hasta el nivel familia o género, dependiendo de la disponibilidad de claves taxonómicas. Finalmente, se registró el número de individuos y los índices de diversidad Alfa $(\alpha)$ y Beta $(\beta)$; abundancia y similitud fueron calculados siguiendo a Moreno (2001).

Para la diversidad Alfa, o grado de diversidad, se calculó tanto la riqueza específica así como los índices de Shannon-Wiener y Simpson.

Para la diversidad Beta, o grado de similaridad, fueron utilizados los índices de Jaccard y Sørensen (Moreno, 2001).

Las diferencias en cuanto a la riqueza, diversidad y abundancia de ácaros entre las dos localidades estudiadas fueron evaluadas mediante análisis de varianza de una vía y las comparaciones fueron hechas mediante pruebas de asociación de $\chi^{2}$ usando el paquete estadístico Statistix versión 8.0. Las curvas de acumulación de especies fueron obtenidas utilizando el programa EstimateS 8 (Colwell, 2006), considerando 100 iteraciones y usando el método de Coleman (Gotelli \& Colwell, 2001)

\section{RESULTADOS Y DISCUSIÓN}

Se colectó un total de 3.466 individuos incluidos en 39 familias y 51 morfotipos de ácaros de los órdenes Prostigmata, Mesostigmata y Astigmata en las dos zonas de muestreo. El mayor número fue colectado en el Matorral donde se encontraron 2.514 individuos en 28 familias y 36 morfoespecies, mientras que en el Bosque Deciduo se encontraron 952 individuos en 33 familias y 43 morfoespecies $\left(\chi^{2}=11,74 ; \mathrm{P}<0,0028 ; \mathrm{gl}=2,1\right)$ (Tab. I). La tendencia de las curvas de acumulación de especies sugiere que el esfuerzo de muestreo fue suficiente para representar el número de especies presentes en ambas localidades (Fig. 1).

En ambas localidades, el número de ácaros Prostigmata fue consistentemente mayor y representó 71,0 y $78,2 \%$ del total colectado en el Matorral y Bosque 
Deciduo, mientras que Mesostigmata constituyó 28,6 y $21,6 \%$ y Astigmata sólo 0,4 y $0,2 \%$, respectivamente (Fig. 2). Posiblemente, la mayor abundancia relativa de ácaros Prostigmata en el suelo pueda ser explicada por la mayor diversidad de hábitos alimenticios de este grupo de ácaros (NeHER \& BARBERCHECK, 1999), lo cual podría permitir un mejor aprovechamiento de los recursos disponibles y por ende ocupar mayor número de nichos.

En el Matorral se encontró un total de 15 familias del orden Prostigmata, siendo Eupodidae la familia con el mayor número de individuos colectados $(70,3 \%)$ seguida de Erythraeidae (7,7\%) y Cunaxidae (6,8\%). De manera similar, se encontraron 16 familias en el Bosque Deciduo y nuevamente el mayor número de individuos colectados fue registrado en Eupodidae (54,9\%), seguido de Erythraeidae (9,8\%), Cunaxidae (9,4\%), Anystidae $(7,0 \%)$ y Trombidiidae $(6,3 \%)$, mientras que la abundancia relativa de las familias menos frecuentes varió entre $0,06-0,2$ y $0,13-1,2 \%$ en el Matorral y Bosque Deciduo, respectivamente. Entre estas, las familias más raras fueron Nanorchestidae, Camerobiidae y Stigmaeidae que sólo fueron encontradas en el Bosque Deciduo.

La abundancia de los ácaros de la familia Eupodidae ha sido registrada tanto en suelos de clima tropical como de clima templado. En Brasil, Mineiro \& Moraes (2002), observaron que Eupodidae fue la familia más abundante en la hojarasca proveniente de un bosque secundario $(34,5 \%)$ y de una plantación de caucho, Hevea brasiliensis (Euphorbiaceae) $(70,6 \%)$, seguidos de Pachygnathidae (20\%), Tetranychidae $(19,3 \%)$ y Rhagidiidae (13,1\%). De manera similar, KALÚz (2005) ratificó que Eupodidae mostró la mayor abundancia en tres ambientes localizados en áreas montañosas de Slovakia con diferentes condiciones de altura y vegetación. Aunque el conocimiento sobre la biología y los hábitos alimenticios de Eupodidae es escaso, se presume que este grupo de ácaros pudiera presentar mayor capacidad para aprovechar los recursos en ambientes que muestran poca diversidad de hojarasca, tal como lo evidencian los resultados obtenidos por Mineiro \& Moraes (2002) y en la presente investigación, donde aproximadamente $70 \%$ de la acarofauna estuvo representada por Eupódidos en ecosistemas de escasa diversidad vegetal.

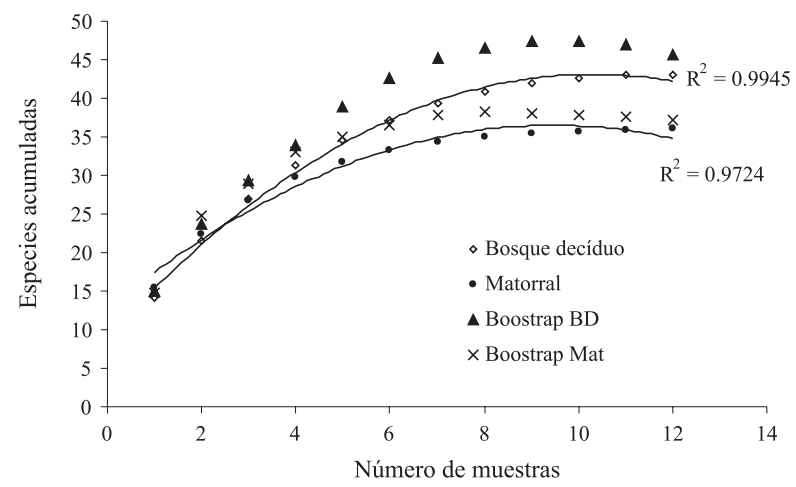

Fig. 1. Curvas de acumulación de especies de ácaros asociados a la hojarasca de un bosque deciduo $(\diamond)$ y matorral $(\bullet)$ del Parque Universitario de la UCLA Lara, Venezuela, diciembre 2000 a noviembre 2001
En el orden Mesostigmata se encontraron 12 familias en el Matorral con predominancia de individuos pertenecientes a Macrochelidae $(80,1 \%)$, seguido de Ameroseiidae, Ologamasidae y Phytoseiidae, mientras que en el Bosque Deciduo, aunque Macrochelidae fue registrada como la más abundante de las 16 familias encontradas, ésta sólo representó 27,5\%, seguido de Parasitidae (20,3\%), Ascidae (15,5\%), Parholaspidae $(9,7 \%)$ y Laelapidae $(9,2 \%)$. Mineiro \& Moraes (2001) consiguieron que la mayor abundancia de ácaros Mesostigmata provenientes de hojarasca de un bosque secundario en São Paulo, Brasil estuvo representada por Ologamasidae $(57,5 \%$ ) y Podocinidae $(9,4 \%)$, mientras que en la hojarasca de caucho se encontró que Laelapidae fue colectada en mayor porcentaje $(26,3 \%)$, seguido de Phytoseiidae $(25,5 \%)$. Probablemente, la mayor abundancia de Macrochelidae observada en el presente estudio este relacionada con el método de muestreo puesto que, con las trampas de caída también fueron colectados una amplia variedad de artrópodos, principalmente insectos, que llevaban estos ácaros sobre su cuerpo debido al hábito forético de Macrochelidae.

Con relación a Astigmata, sólo una familia (Acaridae) fue registrada con porcentajes de 0,4 y $0,2 \%$ en el Matorral y en Bosque Deciduo, respectivamente. De manera similar, Mineiro \& Moraes (2002) también demuestran que los ácaros Astigmata fueron colectados en menor porcentaje tanto en el bosque $(21,2 \%)$ como en plantaciones de caucho $(38,5 \%)$ al ser comparado con Prostigmata. Sin embargo, a diferencia del presente estudio, estos autores registraron mayor diversidad de Astigmata, pues encontraron siete especies en cuatro familias. La escasa presencia de Astigmata en suelos y hojarasca del presente estudio podría ser explicada debido a que estos ácaros han desarrollado relaciones simbióticas con otros organismos (O'CONNOR, 1982), por lo que han evolucionado para explotar otros ambientes diferentes al suelo.

Adicionalmente, las diferencias obtenidas en la composición de familias de ácaros pueden ser debidas a variaciones en el tipo de vegetación, régimen de humedad del suelo e incluso a metodología de muestreo.

La uniformidad de ocurrencia de los diferentes morfotipos de ácaros fue ligeramente mayor en el Bosque

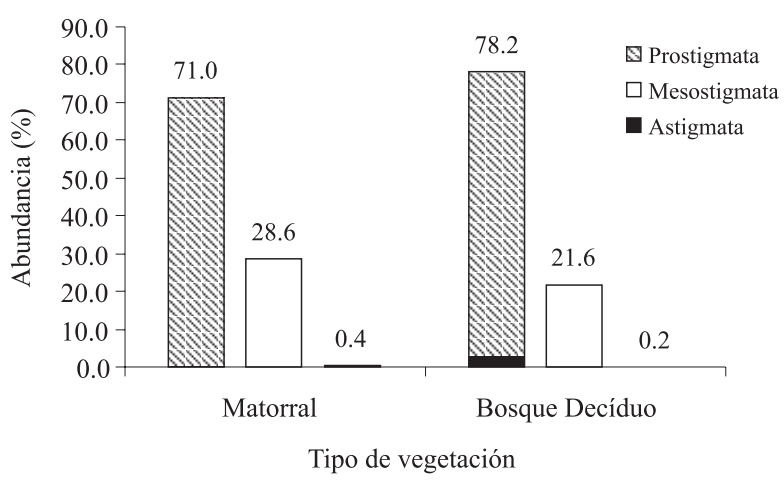

Fig. 2. Abundancia de ácaros (Prostigmata, Mesostigmata y Astigmata) en el Matorral y en el Bosque Deciduo del Parque Universitario de la UCLA, Lara, Venezuela, diciembre 2000 a noviembre 2001. 
Tabla I. Número total de ácaros (Prostigmata, Mesostigmata y Astigmata) asociados a la hojarasca de dos tipos de vegetación del Parque Universitario de la UCLA, Lara, Venezuela, diciembre 2000 a noviembre 2001.

\begin{tabular}{|c|c|c|c|c|}
\hline & \multirow[b]{2}{*}{ Género } & \multicolumn{2}{|c|}{ Tipo de vegetación } & \multirow[b]{2}{*}{$\Sigma$} \\
\hline & & Matorral & Bosque Decíduo & \\
\hline \multicolumn{5}{|l|}{ PROSTIGMATA } \\
\hline Alicorhagididae & Morfotipo 1 & 1 & & 1 \\
\hline Anystidae & Anystis von Heyden, 1826 & 49 & 52 & 101 \\
\hline Bdellidae & Bdella Latreille, 1795 & 52 & 20 & 72 \\
\hline Caeculidae & Morfotipo 2 & & 1 & 1 \\
\hline Camerobiidae & Morfotipo 3 & & 1 & 1 \\
\hline Cheyletidae & Paracheyletia Volgin, 1955 & 2 & 1 & 3 \\
\hline \multirow[t]{2}{*}{ Cunaxidae } & Cunaxa von Heyden, 1826 & 106 & 53 & 159 \\
\hline & Cunaxoides Baker \& Hoffman, 1948 & 16 & 17 & 33 \\
\hline \multirow[t]{5}{*}{ Erythraeidae } & Balaustium von Heyden, 1826 & & 17 & 17 \\
\hline & Caeculisoma Berlese, 1888 & & 1 & 1 \\
\hline & Callidosoma Womersley, 1936 & 2 & 1 & 3 \\
\hline & Erythraeus Latreille, 1806 & 128 & 53 & 181 \\
\hline & Leptus Latreille, 1796 & 8 & 1 & 9 \\
\hline Eupalopsellidae & Morfotipo 4 & 4 & & 4 \\
\hline \multirow[t]{2}{*}{ Eupodidae } & Eupodes Koch, 1835 & 608 & 161 & 769 \\
\hline & Linopodes Koch, 1835 & 646 & 247 & 893 \\
\hline Nanorchestidae & Morfotipo 5 & & 1 & 1 \\
\hline Penthalodidae & Morfotipo 6 & 2 & 1 & 3 \\
\hline Rhagidiidae & Morfotipo 7 & 3 & 1 & 4 \\
\hline \multirow[t]{2}{*}{ Smariidae } & Hirstiosoma Womersley, 1934 & & 9 & 9 \\
\hline & Smaris Latreille, 1796 & 15 & 24 & 39 \\
\hline Stigmaeidae & Morfotipo 8 & & 1 & 1 \\
\hline Tarsonemidae & Poliphagotarsonemus & 1 & & 1 \\
\hline \multirow[t]{2}{*}{ Tetranychidae } & Mononychellus Wainstein, 1971 & & 1 & 1 \\
\hline & Tetranychus Dufour, 1832 & 16 & 17 & 33 \\
\hline Trombidiidae & Trombidium Fabricius, 1775 & 54 & 47 & 101 \\
\hline \multirow[t]{3}{*}{ Tydeidae } & Lorryia Oudemans, 1925 & 2 & 1 & 3 \\
\hline & Prolorryia Oudemans, 1924 & 2 & & 2 \\
\hline & Tydeus Koch, 1836 & 68 & 14 & 82 \\
\hline Sub-total & & 1.785 & 743 & 2.528 \\
\hline \multicolumn{5}{|l|}{ MESOSTIGMATA } \\
\hline Ameroseiidae & Morfotipo 9 & 38 & 4 & 42 \\
\hline Ascidae & Morfotipo 10 & 7 & 32 & 39 \\
\hline Digamasellidae & Morfotipo 11 & 9 & & 9 \\
\hline Discozerconidae & Morfotipo 12 & & 1 & 1 \\
\hline Heterozerconidae & Morfotipo 13 & 5 & & 5 \\
\hline Ichthyostomatogasteridae & Morfotipo 14 & 4 & 2 & 6 \\
\hline Laelapidae & Morfotipo 15 & 16 & 19 & 35 \\
\hline Macrochelidae & Macrocheles Latreille, 1829 & 576 & 57 & 633 \\
\hline Macronyssidae & Morfotipo 16 & & 1 & 1 \\
\hline Ologamasidae & Morfotipo 17 & 32 & 6 & 38 \\
\hline Pachylaelapidae & Morfotipo 18 & & 5 & 5 \\
\hline Parasitidae & Morfotipo 19 & & 42 & 42 \\
\hline Parholaspidae & Morfotipo 20 & & 20 & 20 \\
\hline \multirow[t]{2}{*}{ Phytoseiidae } & Euseius Wainstein, 1962 & 2 & 3 & 5 \\
\hline & Proprioseiopsis Muma, 1961 & 22 & 1 & 23 \\
\hline Polyaspidae & Morfotipo 21 & 1 & & 1 \\
\hline Rhynonissidae & Morfotipo 22 & & 1 & 1 \\
\hline Uropodidae & Oodinychus Berlese, 1918 & 5 & 4 & 9 \\
\hline Vegaiidae & Morfotipo 23 & 2 & 2 & 4 \\
\hline Zerconidae & Morfotipo 24 & & 7 & 7 \\
\hline Sub-total & & 719 & 207 & 926 \\
\hline \multicolumn{5}{|l|}{ ASTIGMATA } \\
\hline \multirow[t]{2}{*}{ Acaridae } & Acarus Linnaeus, 1758 & 7 & & 7 \\
\hline & Tyrophagus Oudemans, 1923 & 3 & 2 & 5 \\
\hline Sub-total & & 10 & 2 & 12 \\
\hline Total General & & 2.514 & 952 & 3.466 \\
\hline
\end{tabular}


Deciduo, lo cual fue consistente con el valor del índice de Shannon, por lo que se considera que el tipo de vegetación predominante en esta zona de muestreo permite albergar mayor diversidad de ácaros cuando se compara con el Matorral (Tab. II). Las diferencias observadas entre ambas localidades pueden ser explicadas principalmente por la ocurrencia de variaciones en las propiedades del suelo, incluyendo el régimen de humedad como efecto de la cobertura vegetal, lo cual tiene incidencia directa sobre las poblaciones de microartrópodos (KenNedy, 1999). Adicionalmente, la riqueza y diversidad de plantas también pueden influir la riqueza y diversidad faunística mediante mecanismos que alteran la cantidad, calidad y heterogeneidad de los recursos disponibles para la fauna del suelo ( $\mathrm{S}_{\mathrm{T}}$. JoHn et al., 2006). SiLva et al. (2004) afirmaron que la mayor diversidad de ácaros pudiera estar correlacionada con el espesor y diversidad de la hojarasca a partir de la cual éstos fueron muestreados. Por otra parte, el Bosque Deciduo puede constituir un hábitat más estable dado que la vegetación predominante está conformada principalmente por árboles de más de $5 \mathrm{~m}$ de altura, cuyo aporte de materia orgánica es más alto pudiendo albergar un mayor número de taxa. Mineiro \& Moraes (2002) encontraron que la diversidad de ácaros Prostigmata y Astigmata era ligeramente mayor en hojarasca proveniente de un bosque $\left(\mathrm{H}^{\prime}=2,24\right)$ comparado con hojarasca de una plantación de caucho $\left(H^{\prime}=2,22\right)$. Contrariamente, Mineiro \& Moraes (2001) registraron la mayor diversidad de Mesostigmata en la hojarasca proveniente de la plantación de caucho $\left(\mathrm{H}^{\prime}=2,30\right)$ comparada con la diversidad encontrada en el bosque secundario $\left(H^{\prime}=2,26\right)$. Consistentemente, se demostró que ambas localidades presentan sólo 10 y 14 especies comunes, lo cual se refleja en un bajo grado de similitud tal como lo revela el índice de Jaccard (J') de 0,333 y 0,350 (Mineiro \& Moraes, 2001, 2002).

A pesar que se observó mayor diversidad en el Bosque Deciduo, se encontró mayor dominancia en el Matorral, tomando como base el índice de Simpson (Tab. II). Esto se explica porque del total colectado en el Matorral, el mayor porcentaje $(72,8 \%)$ de los especimenes

Tabla II. Índices de diversidad, abundancia y similitud de ácaros Prostigmata, Mesostigmata y Astigmata asociados a hojarasca de dos tipos de vegetación del Parque Universitario de la UCLA, Lara, Venezuela, diciembre 2000 a noviembre 2001.

\begin{tabular}{lrc}
\hline & \multicolumn{2}{c}{ Formación Vegetal } \\
\hline & Matorral & Bosque Deciduo \\
Número de individuos & 2.514 & 952 \\
Uniformidad (E) & 0,52 & 0,69 \\
Riqueza ( $S$ ) & 36 & 43 \\
Índice de Shannon (H') & 2,12 & 2,67 \\
Varianza de Shannon & 0,00065 & 0,00156 \\
Índice de Simpson (Dsp) & 0,18374 & 0,11665 \\
1/DSp & 5,44 & 8,57292 \\
Número de morfotipos comunes & \multicolumn{2}{c}{28} \\
Número de familias comunes & \multicolumn{2}{c}{22} \\
Índice de Jaccard $(\mathrm{J}$ ') & \multicolumn{2}{c}{0,59} \\
Indice de Sørensen ( $\mathrm{I}_{\mathrm{s}}$ ) & \multicolumn{2}{c}{0,74} \\
\hline
\end{tabular}

pertenecía a las familias Eupodidae y Macrochelidae, mientras que en el Bosque Deciduo estas mismas familias estuvieron representadas sólo en un $48,5 \%$. Adicionalmente, la uniformidad de las curvas de frecuencia de ocurrencia fue mayor en el Bosque Deciduo que en el Matorral, tal como es evidenciado por la menor pendiente de la primera (Fig. 3). Estas curvas pueden ser utilizadas para evaluar el grado de disturbancia de una comunidad (SiLva et al., 2004), por lo que se presume que el Bosque Deciduo representa un hábitat menos disturbado que el Matorral. Finalmente, en áreas donde existen mayor cantidad de factores estresantes, la diversidad tiende a ser más baja y la abundancia de la especie más común tiende a incrementarse mientras que la abundancia de las especies más raras tiende a disminuir hasta el punto en el cual algunas especies raras pueden extinguirse (ODum, 1988).

Finalmente, se encontró que de las 51 morfoespecies de ácaros encontradas, 28 de ellas estuvieron representadas en ambos sitios de muestreo, lo cual permitió establecer un grado de similitud moderado, basándose en los índices de similitud de Jaccard $\left(\mathrm{J}^{\prime}=0,59\right)$ y Sørensen $\left(\mathrm{I}_{\mathrm{s}}=0,70\right)$ (Tab. II). De acuerdo con Moreno (2001), el intervalo de valores para estos índices va de 0 , cuando no hay especies compartidas entre ambos sitios, hasta 1 cuando los dos sitios tienen la misma composición de especies. Este valor de similitud puede ser debido a la proximidad entre ambas zonas de muestreo, lo que permite que algunas familias se adapten a las condiciones del Matorral y Bosque deciduo.

Este estudio constituye el primero en abordar la diversidad de ácaros Prostigmata, Mesostigmata y Astigmata asociados a la hojarasca en el Parque Universitario de la UCLA y evidenció una amplia diversidad de este importante grupo de artrópodos, sobre todo en el Bosque Deciduo, donde posiblemente el mayor aporte de materia orgánica permitiría albergar mayor número de morfotipos, con diferentes hábitos alimenticios. Sin embargo, se sugiere realizar estudios taxonómicos más detallados, que incluyan los ácaros Cryptostigmata, para logar un mejor conocimiento sobre

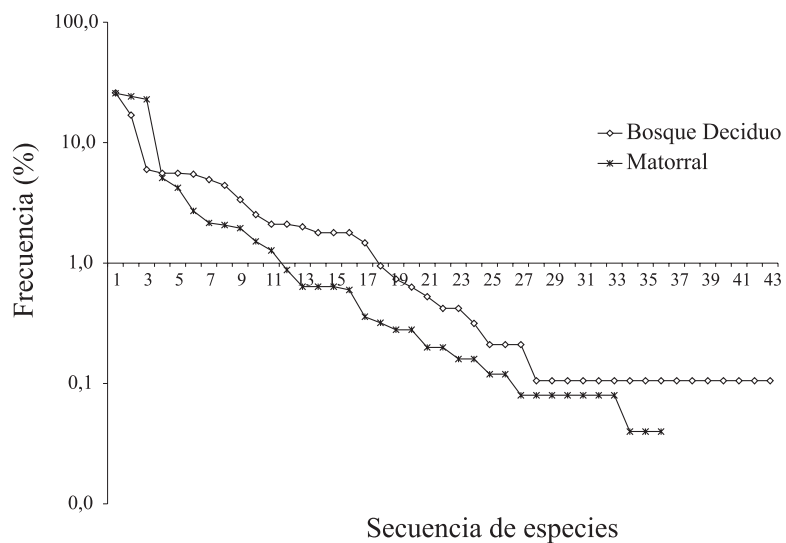

Fig. 3. Curva de frecuencia de ocurrencia de los ácaros (Prostigmata, Mesostigmata y Astigmata) asociados al suelo en el Matorral y en el Bosque Deciduo del Parque Universitario de la UCLA, Lara, Venezuela, diciembre 2000 a noviembre 2001. 
la acarofauna de la región centroccidental de Venezuela. Por otra parte, la marcada abundancia de los géneros Eupodes y Linopodes (Eupodidae) y Macrocheles (Macrochelidae) sugiere la necesidad de realizar estudios futuros que incluyan observaciones biológicas de estos y así determinar su posible rol en el mantenimiento de los ecosistemas en suelos de las regiones tropicales.

Agradecimientos. A la Prof. Lisbeth Díaz, ao Prof. Javier Lorbes (Universidad Centroccidental Lisandro Alvarado) por la asesoría en el análisis estadístico. Al Consejo de Desarrollo Científico, Humanístico y Tecnología (CDCHT-UCLA) por el financiamiento parcial del proyecto 004-TAG-2004.

\section{REFERENCIAS BIBLIOGRÁFICAS}

Anderson, J. M. 1978. Inter- and intra-habitat relationships between woodland Cryptostigmata species diversity and diversity of soil and microhabitats. Oecologia 32(3):341-348.

Balogh, J. 1972. The Oribatid genera of the World. Budapest, Akademiai Kiado. 331p.

BAlogh, J. \& BAlOGH, P. 1988. Oribatid mites of the Neotropical Region I. Soil mites of the World 2. Budapest, Akademiai Kiado. 335p.

1990. Oribatid mites of the Neotropical Region II. Soil mites of the World 3. Budapest, Akademiai Kiado. 333p.

Behan-Pelletier, V.; Paoletti, M.; Bissett, B. \& Stinner, B. B. 1993. Oribatid mites of forest habitants in northern Venezuela. Tropical Zoology 1(Special Issue):39-54.

Bracho, A.; Contreras, M.; Villalobos, Y.; Bracho, B.; Quirós, M.; JimÉneZ , L. \& LARreAl, M. 1999. Cambios en la cantidad y la biodiversidad de la mesofauna en un suelo degradado con aplicación de abono orgánico. Revista de la Facultad de Agronomía 16(Supl. 1):187-195.

Brown, G. G. \& Fragoso, C. 2003. El uso de la macrofauna edáfica en la agricultura del siglo XXI: problemas y perspectivas. In: Brown, G. G., Fragoso, C. \& Oliveira, L. J. eds. O uso da macrofauna edáfica na agricultura do século XXI: a importância dos engenheiros do solo. Londrina, EMBRAPA (Documentos 224). p.11-19.

Colwell, R. K. 2006. EstimateS: statistical estimation of species richness and shared species from samples. Version 8.0. Disponible en: < http//www.purl.oclc.org.estimates>. Acceso en: 14.04.2007.

Gaston, K. J. 1992. Regional numbers of insect and plant species. Functional Ecology 6(3):243-247.

Gotelli, N. J \& Colwell, R. K. 2001. Quantifying biodiversity: procedures and pitfalls in the measurement and comparison of species richness. Ecology Letter 4:379-391.

Hättenschwiler, S.; Tiunov, A. V. \& Scheu, S. 2005. Biodiversity and litter decomposition in terrestrial ecosystems. Annual Review of Ecology, Evolution and Systematics 36:191-218.

Herderson, R. 2001. Technique for positional slide-mounting of Acari. Systematic and Applied Acarology Special Publications 7:1-4. Disponible en: <www.nhm.ac.uk/ hosted_sites/acarology/saas/saasp.html>. Acceso en: 14.04.2007.
Holdridge, L. R. 1979. Life Zone Ecology. San José, Tropical Science Center. $216 \mathrm{p}$.

KaLúz, S. 2005. Soil and soil mites (Acari) of the Ski slope in Nitke Trati Mts. Ekológia 24(2):200-213.

Kennedy, A. 1999. Microbial Diversity in Agroecosystem Quality. In: Collins, W. \& Qualset, C. eds. Biodiversity in Agroecosystems. Washington, CRC. p.1-17.

Krantz, G. 1978. A manual of Acarology. Corvallis, Oregon State University. 509p.

López, J. 1995. Caracterización físico natural del Parque Universitario de la Universidad Centroccidental Lisandro Alvarado. Bioagro 7(3):85-90.

MadeJ, G. \& Skubala, P. 2002. Colonization of a dolomitic dump by mesostigmatid mites (Acari: Mesostigmata). In: Bernini, F.; Nanneli, R.; Nuzzaci, G. \& De Lillo, E. eds. Acarid phylogeny and evolution. Adaptations in mites and ticks. Proceedings of the IV symposium of the European Association of Acarologists. Dordrecht, Kluwer Academic. p.175-184.

Mineiro, J. \& Moraes, G. 2001. Gamasida (Arachnida: Acari) edáficos de Piracicaba, Estado de São Paulo. Neotropical Entomology 30(3):379-385.

. 2002. Actinedida e Acaridida (Arachnida: Acari) edáficos de Piracicaba, Estado de São Paulo. Neotropical Entomology 31(1):67-73.

Moreno, C. E. 2001. Métodos para medir la biodiversidad Zaragoza, M\&T-Manuales y Tesis SEA. v.1, p.84.

Neher, D. A. \& Barbercheck, M. E. 1999. Diversity and function of soil mesofauna, In: Collins, W. \& Qualset, C. eds. Biodiversity in Agroecosystems. Washington, CRC. p.27-47.

O'Connor, B. M. 1982. Evolutionary ecology of astigmatid mites. Annual Review of Entomology 27: 385-409.

Odum, E. P. 1988. Ecologia. Rio de Janeiro, Koogan. 434p.

Paoletti, M.; Dreon, A.; Stinner, B. B. \& Stinner, D. 1988 Distribuzione della pedofauna e nutrienti nella selva neotropicale. La foresta di nubi venezuelana. Thalassia Salentina 18:511-531.

Paoletti, M. G.; Taylor, R. A. J.; Stinner, B. R.; Stinner, D. H. \& Benzing, D. H. 1991. Diversity of soil fauna in the canopy and forest floor of a venezuelan cloud forest. Journal of Tropical Ecology 7:373-383.

Petersen, H. 1982. Structure and size of animal soil populations. Oikos 39:306-329.

Prieto, D.; González, V. \& Tcherva, T. 2005. Microartrópodos asociados a la hojarasca de un bosque semidecíduo de Bacunayagua, Matanzas, Cuba. Revista Biología 19(1-2):57-65.

Silva, E.; Moraes, G. \& Krantz, G. 2004. Diversity of edaphic rhodocaroid mites (Acari: Mesostigmata: Rhodacaroidea) in natural ecosystems in the state of São Paulo, Brazil. Neotropical Entomology 33(5):547-555.

St. John, M.; Wall, D. \& Behan-Pelletier, V. M. 2006. Does plant species co-occurrence influence soil mite diversity? Ecologia 87(3):625-633.

Vu, Q. M. \& NGUYen, T. T. 2000. Microarthropod community structures (Oribatei and Collembola) in Tam Dao National Park, Vietnam. Journal of Bioscience 25(4):379-386.

Walter, D. \& Proctor, H. 1999. Mites: ecology, evolution and behaviour. Oxon, CABI. 322p.

Recebido em junho de 2006. Aceito em maio de 2007. ISSN 0073-4721

Artigo disponível em: www.scielo.br/isz 